\title{
The Acceleration of Electrons at Perpendicular Shocks and its Implication for Solar Energetic Particle events
}

\author{
Fan Guo and Joe Giacalone \\ Department of Planetary Sciences, University of Arizona, Tucson, AZ 85721
}

\begin{abstract}
We present a study of the acceleration of electrons at a perpendicular shock that propagates through a turbulent magnetic field. The energization process of electrons is investigated by utilizing a combination of hybrid (kinetic ions and fluid electron) simulations and test-particle electron simulations. In this method, the motions of the test-particle electrons are numerically integrated in the time-dependent electric and magnetic fields generated by two-dimensional hybrid simulations. We show that large-scale magnetic fluctuations effect electrons in a number of ways and lead to efficient and rapid energization at the shock front. Since the electrons mainly follow along magnetic lines of force, the large-scale braiding of field lines in space allows the fast-moving electrons to interact with the shock front and get accelerated multiple times. Ripples in the shock front occurring at various scales will also contribute to the acceleration by mirroring the electrons. Our calculation shows that this process favors electron acceleration at perpendicular shocks. The acceleration efficiency is critically dependent on the turbulence amplitude and coherence length. We also discuss the implication of this study for solar energetic particles (SEPs) by comparing the acceleration of electrons with that of protons. Their correlation indicates that perpendicular shocks play an important role in SEP events.
\end{abstract}

Keywords: acceleration of particles, cosmic rays, shock waves, turbulence

PACS: $96.50 . \mathrm{Pw}$, 96.50.Tf, 96.50.Vg, 52.35.Tc, 52.65.Ww

\section{INTRODUCTION}

The acceleration of charged particles at collisionless shocks is a remarkable process in astrophysics and space physics. The diffusive shock acceleration (DSA) has been very successful in explaining the acceleration process and is thought to be the mechanism for accelerating solar energetic particles (SEPs). However, how a population of low energy particles gain energy, the so-called "injection problem", is still not well understood. The acceleration of electrons at collisionless shocks is considered to be more difficult than that of ions. This is primarily due to the fact that the gyroradii of electrons are much smaller compared with that of protons at the same energy (by a factor of $\sqrt{m_{i} / m_{e}} \sim 43$ ), therefore low energy electrons can not resonantly interact with large-scale turbulence or ion-scale waves. In contrast, energetic electrons are often observed to be associated with quasi-perpendicular shocks.

In SEP events, energetic electrons are found to be associated with energetic ions. Haggerty \& Roelof [1] reported that energetic electrons (175-315 keV) are well associated with protons with energy between 1.8 and $4.7 \mathrm{MeV}$. Cliver [2] showed that, the electrons $(\sim 0.5 \mathrm{MeV})$ and the protons $(>10 \mathrm{MeV})$ in large SEP events are strongly correlated. The correlation still exists even when the isotope ratio $(\mathrm{Fe} / \mathrm{O})$ changes. The tight cor- 
relation between electrons and ions has also been found in ground base level events (Tylka, private communication [3]), where both electrons and protons are accelerated to relativistic energies. It is interesting to note that the high energy electrons and protons are also closely correlated in solar flares [4]. These observations indicate that a common mechanism may exist in the process of particle acceleration during SEP events.

Particle acceleration at perpendicular shocks has been considered as a fast and efficient process $[5,6]$. Giacalone [7, 8] has performed numerical simulations for the acceleration of protons at fast shocks that propagates through a plasma that contains large scale magnetic fluctuations. The acceleration to high energy is very efficient, which indicates that there is no injection problem. Recently, Jokipii \& Giacalone [9] have proposed a mechanism to solve the injection problem that does not require strong pitch-angle scattering from small-scale fluctuations. The fast-moving electrons can move along meandering magnetic field lines and travel back and forth across the shock front, and, therefore gain energy from the difference between the upstream and downstream flow velocities. Using self-consistent hybrid simulations combined with test-particle simulations for electrons, Guo \& Giacalone [10] have found efficient electron acceleration at perpendicular shocks moving through a plasma containing large-scale pre-existing upstream magnetic turbulence. The turbulent magnetic field leads to field-line meandering that allows the electrons to get accelerated at the shock front multiple times, like in the Jokipii \& Giacalone picture. In a more recent paper, Guo \& Giacalone [11] demonstrated that in solar flare region, a low Mach number shock can also efficiently accelerate both electrons and ions. In this paper, we present the results of the acceleration of electrons at a perpendicular shock that propagates through a turbulent magnetic field. The energization process of electrons is investigated by utilizing a combination of hybrid simulations and test-particle electron simulations. We also discuss the implication of this study for SEPs by comparing the acceleration of electrons with that of protons. Their correlation indicates that perpendicular shocks play an important role in SEP events.

\section{NUMERICAL METHOD}

We implement a combination of a 2-D hybrid simulation to model the fields and plasma flow and a test particle simulation to follow the orbits of electrons. In the first step, we employ a two-dimensional hybrid simulation that includes pre-existing large-scale turbulence [8]. In the hybrid simulation model, the ions are treated fully kinetically and thermal (i.e., non-energetic) electrons are treated as a massless fluid. This approach is well suited to resolve ion-scale plasma physics that is critical to describe supercritical collisionless shocks. We consider a two-dimensional Cartesian grid in the $x-z$ plane. All the physical vector quantities have components in three directions, but depend spatially only on the two variables. A shock is produced by continuously injecting plasma from one end $(x=0)$ of the simulation box, and reflected elastically at the other end $\left(x=L_{x}\right)$. This boundary is also assumed to be a perfectly conducting barrier. The pileup of density and magnetic field creates a shock propagating in the $-x$ direction. To include the large-scale magnetic fluctuations, a random magnetic field is superposed on a mean field at the beginning of the simulation and is also injected continuously at the $x=0$ boundary during the simulation. The simplified one-dimensional fluctuations have the 
form $\mathbf{B}(z, t)=\delta \mathbf{B}(z, t)+\mathbf{B}_{1}$, where $\mathbf{B}_{1}$ is the averaged upstream magnetic field. The fluctuating component contains an equal mixture of right- and left-hand circularly polarized, forward and backward parallel-propagating plane Alfven waves. The amplitude of the fluctuations is determined from a Kolmogorov-like power spectrum:

$$
P(k) \propto \frac{1}{1+\left(k L_{c}\right)^{5 / 3}},
$$

in which $L_{c}$ is the coherence scale of the fluctuations. For the simulations presented in this study, we take $L_{c}=L_{z}$, where $L_{z}$ is the size of simulation box in the $z$ direction. As a standard case of this study, we consider a turbulence variance $\sigma=\delta B^{2} / B_{1}^{2}=0.3$. The size of the simulation box for most of situations is $L_{x} \times L_{z}=400 c / \omega_{p i} \times 1024 c / \omega_{p i}$, where $c / \omega_{p i}$ is the ion inertial length. The Mach number of the flow in the simulation frame is $M_{A 0}=4.0$, and the averaged Mach number in the shock frame is about 5.6. The averaged shock normal angle $\left\langle\theta_{B n}\right\rangle=90^{\circ}$, but we also present the cases for $\left\langle\theta_{B n}\right\rangle=60^{\circ}$ and $75^{\circ}$. We also discuss the effect of different values of turbulence variances and correlation lengths. The other important simulation parameters include electron and ion plasma beta $\beta_{e}=0.5$ and $\beta_{i}=0.5$, respectively, grid sizes $\Delta x=\Delta z=0.5 c / \omega_{p i}$, time step $\Delta t=0.01 \Omega_{c i}^{-1}$, the ratio between light speed and upstream Alfven speed $c / v_{A 1}=8696.0$, and the anomalous resistivity is taken to be $\eta=1 \times 10^{-5} 4 \pi \omega_{p i}^{-1}$. The initial spatially uniform thermal ion distribution was generated using 40 particles per cell. Under these parameters, the upstream Alfven speed is about $34.5 \mathrm{~km} / \mathrm{s}$ and the shock speed is about $193 \mathrm{~km} / \mathrm{s}$ in upstream frame.

In the second part of our calculation we integrate the motion equation of an ensemble of test-particle electrons in the electric and magnetic fields obtained in the hybrid simulations. We assume non-relativistic motions, which is reasonable because the highest energy of electrons in our study is still non-relativistic. We release a shell distribution of electrons with energy of $100 \mathrm{eV}$, which corresponds to an electron velocity $V_{e}=30.7 U_{1}=5.7 v_{\text {the }}$ in the upstream frame, where $U_{1}$ is upstream bulk velocity in the shock frame and $v_{\text {the }}$ is the thermal velocity of fluid electrons considered in the hybrid simulations, respectively. We use a second-order spatial interpolation and a linear temporal interpolation, which ensure the smooth variations of the electromagnetic fields. The test-particle electrons are released uniformly upstream when the shock has fully formed and is far from the boundaries. The numerical technique used to integrate electron trajectories is the so-called Bulirsh-Stoer method. It is highly accurate and conserves energy well. It is fast when fields are smooth compared with the electron gyroradius. The algorithm uses an adjustable time-step method based on the evaluation of the local truncation error. The time step is allowed to vary between $5 \times 10^{-4}$ and $0.1 \Omega_{c e}^{-1}$, where $\Omega_{c e}$ is the electron gyrofrequency. The ratio $\Omega_{c e} / \Omega_{c i}$ is taken to be the realistic value 1836 . The total number of electrons in the simulation is $1.6 \times 10^{6}$. The electrons that reach the left or right boundary are assumed to escape from the shock region and are removed from the simulation. The boundary condition in the $z$ direction is taken to be periodic. The readers are referred to $[12,10]$ for more details on the numerical methods. 


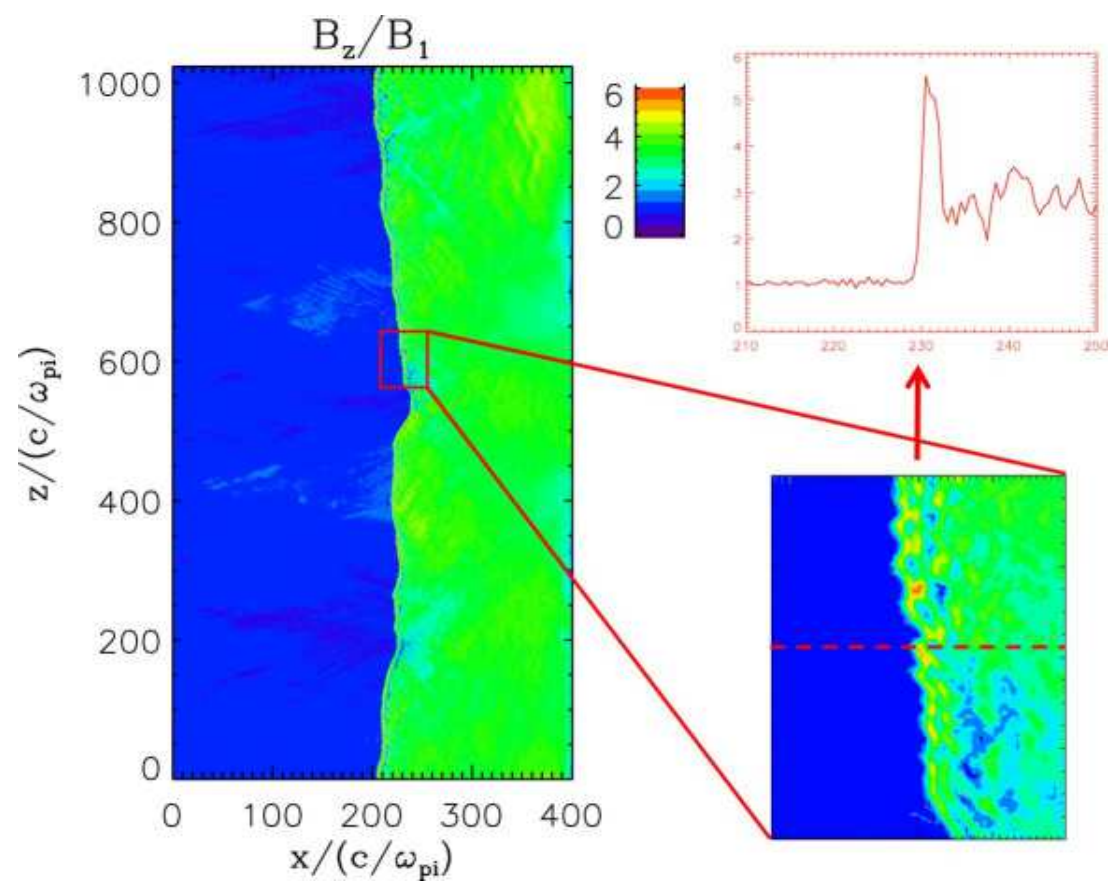

FIGURE 1. A snapshot of the magnetic field in the z-direction $B_{z} / B_{1}$ represented in color-coded scale at $t=110 \Omega_{c i}^{-1}$, where $B_{1}$ is the averaged upstream magnetic field strength. A region at the shock front is zoomed in on the right bottom and a profile is illustrated on the right upper panel. The shock surface is shown to be rippled and irregular in different scales.

\section{SIMULATION RESULTS}

Figure 1 shows a snapshot of the $z$ component of the magnetic field, $B_{z} / B_{1}$, at $t=$ $110 \Omega_{c i}^{-1}$. At this time, the shock is fully developed. The position of the shock front is clearly seen from the boundary of the magnetic field jump. On the right bottom a small region of the simulation domain is zoomed in, which shows small scale irregularities at the shock front. Because of the effect of the large-scale turbulence, the shock surface become irregular on a variety of spatial scales from small-scale ripples to large-scale structures caused by the interaction between the shock and the upstream turbulence $[7,13,10]$. Locally, the structure of the shock, shown in top right panel, is still clearly a quasi-perpendicular shock. The meandering of field lines close to the shock surface helps to trap the electrons at the shock, leading to efficient acceleration. The shock ripples also contribute to the acceleration by mirroring electrons between them. This is shown by examining the trajectories of some electrons as illustrated in Figure 2. In this figure, the top left plot displays the trajectory of a representative electron in the $x-z$ plane, overlapped with a 2-D gray-scale representation of $B_{z}$ at $\Omega_{c i} t=89.0$. The upper right plot shows the position of this electron (in $x$ ) as a function of time. The electron bounces back and forth between the ripples for several times. The energy change as a function of the $x$ position, corresponding to these reflections is shown in the bottom left panel. We find that there are jumps in energy at each of the reflections. The panel on the bottom right shows the electron energy as a function of time that also illustrates the 

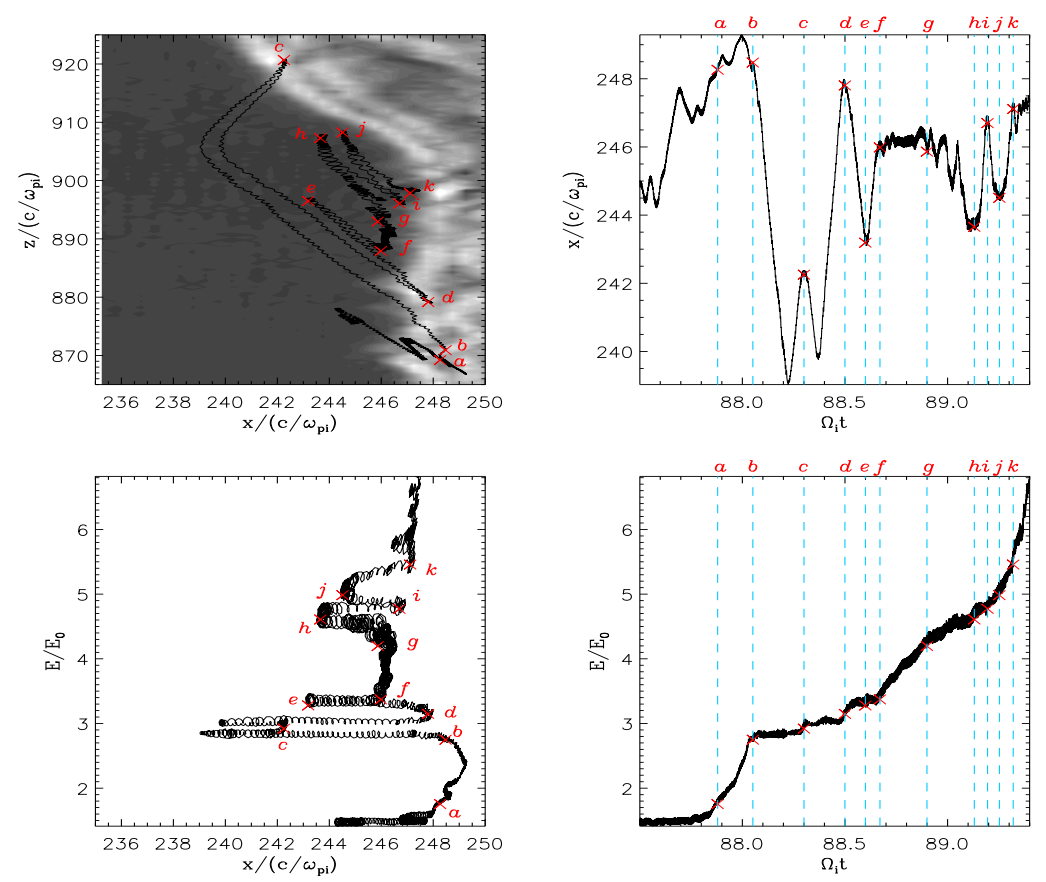

FIGURE 2. An electron trajectory analysis. The top left panel shows the trajectory of the electron in the $x-z$ plane, overlapped with a contour of $B_{z}$ magnetic field; The top right panel shows the position of the electron in the $x$ direction as a function of time; The bottom left panel illustrates the electron energy $E / E_{0}$ as a function of $x$; The bottom right panel shows the dependence of electron energy $E / E_{0}$ on time.

features of multiple accelerations related to the multiple reflections. Since the electrons mainly follow along the magnetic lines of force, the large-scale braiding of field lines in space allows the fast-moving electrons to interact with the shock front multiple times. The electrons can also travel along the meandering magnetic field and gain energy from the velocity difference between upstream and downstream [9].

We now discuss the effect of varying the averaged shock normal angle, the value of turbulence variances, and the values of correlation lengths. In the top panels of Figure 3 we present the electron energy spectra for left: three different mean shock-normal angles $\left(\left\langle\theta_{B n}\right\rangle=60^{\circ}, 75^{\circ}\right.$ and $90^{\circ}$, respectively), middle: three cases with different turbulence variances $\sigma=0.1,0.3$, and 0.5 , respectively, and right: three cases with different sizes of the simulation box and correlation length $L_{z}=2000,1024$, and $400 c / \omega_{p i}$. All the spectra are obtained in the end of the simulation $\Omega_{c i} t=120$ and do not change any more. We find this process prefer a perpendicular shock, in which more electrons are accelerated to high energy. It is also found that the energy spectra harden at high energies when the turbulence variance and/or turbulence correlation length is larger, which indicates that the large-scale turbulence is important for accelerating electrons to high energies. The reason is that the meandering of field lines is enhanced, which allows the electrons have a better chance to travel though the shock multiple times.

We explicitly compare the energy spectra of electrons with that of protons. In the bottom panels of Figure 3 we show the energy spectra of downstream protons from the hybrid simulations, corresponding to the spectra of electrons in the top panels. We find 

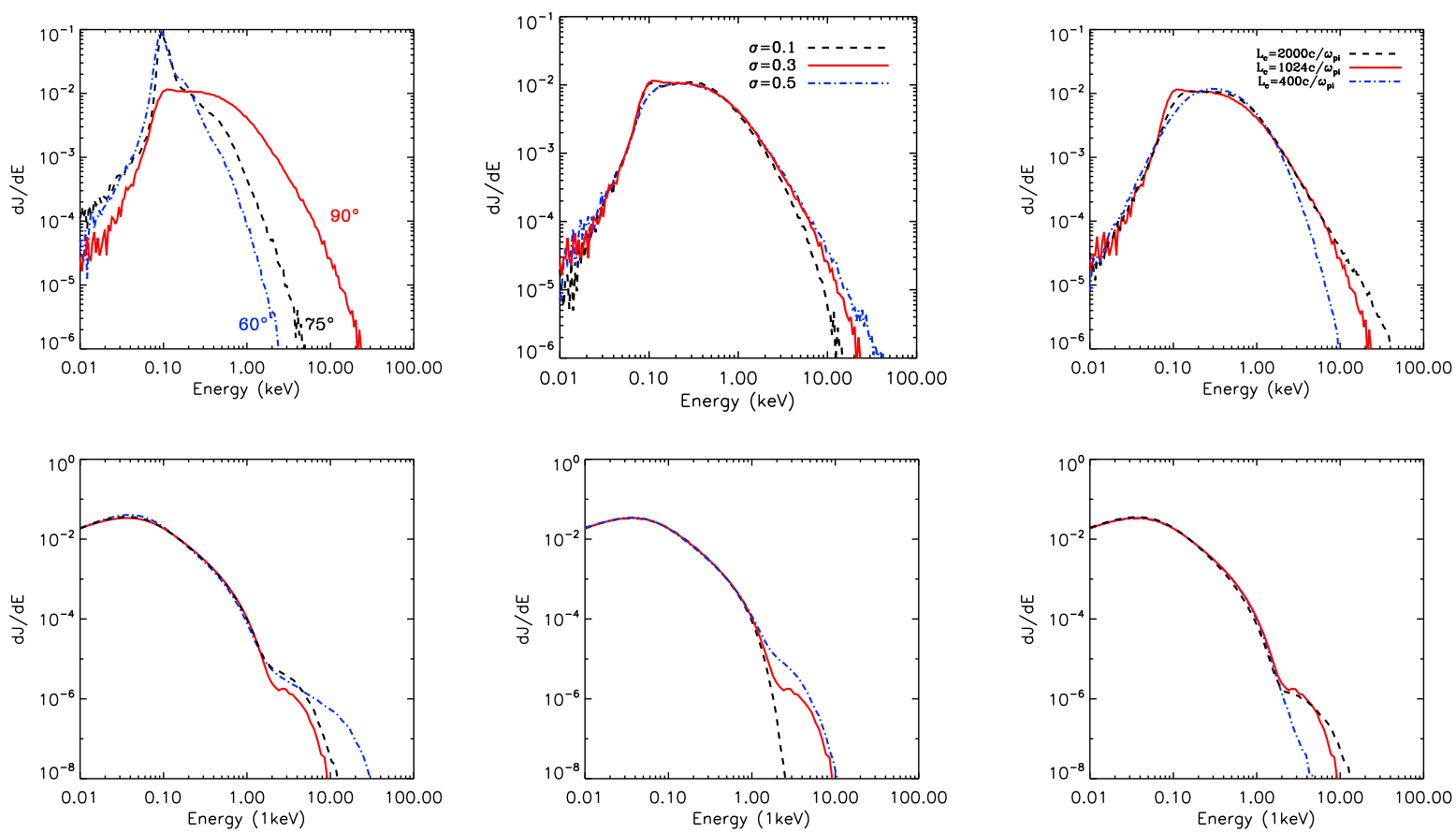

FIGURE 3. Top: energy spectra of accelerated electrons from test-particle electron simulations. Bottom: The energy spectra of protons directly from hybrid simulations. See description in the text for details.

more efficient acceleration for protons can be obtained in the case of larger values of turbulence variances and correlation lengths. These agree well with the characteristics of the acceleration of electrons. In our simulations, the accelerated protons at the oblique shock with $\left\langle\theta_{B n}\right\rangle=60^{\circ}$ are found to reach higher energies, which is different than previous works $[5,6,7,8]$. This is probably due to the limited temporal and spatial scales of our simulations. As shown by Giacalone [8] using test-particle simulations, the energy spectra of protons reach the highest energy in perpendicular shock case in a longer time scale $\Omega_{c i} t \sim 50000$ (this corresponds to $5-10$ minutes for typical parameters in solar corona). However the current results from hybrid simulations do show a population of thermal protons can be accelerated to high energies in perpendicular shocks, which supports the idea that both electrons and protons can be efficiently accelerated by shocks with large shock normal angles.

\section{SUMMARY}

We have presented the results of the acceleration of electrons (and also protons) at a perpendicular shock that propagates through a turbulent magnetic field. The acceleration of electrons are enhanced due to the effect of large-scale magnetic turbulence. The accompanying results for protons qualitatively show the correlation between accelerated electrons and accelerated ions in oblique shocks with large shock normal angles. Since the tight correlation between electrons and ions has been observed in SEP events. This 
result indicates that quasi-perpendicular/perpendicular shocks play an important role in SEP events.

\section{ACKNOWLEDGMENTS}

The authors thank Dr. J. R. Jokipii and Dr. Jozsef Kota for valuable discussions and Dr. David Burgess for sharing the details about the simulations method. The authors also thank Dr. Ed Cliver, Dr. Dennis Haggerty and Dr. Allan Tylka for discussions and informations regarding the observations of SEP events related to this project. This work was supported by NASA under grants NNX10AF24G and NNX11AO64G.

\section{REFERENCES}

1. Haggerty, D. K., \& Roelof, E. C. 2009, in AIP Conf. Proc. 1183, Shock waves in Space and Astrophysical Environments, ed. X. Ao, R. H. Burrows, \& G. P. Zank (Melville, NY: AIP), 3

2. Cliver, E. W. 2009, Central European Astrophysical Bulletin, 33, 253

3. Tylka, A. J., private communication in March 2012.

4. Shih, A. Y., Lin, R. P., \& Smith, D. M. 2009, The Astrophysical Journal, 698, L152

5. Jokipii, J. R. 1982, The Astrophysical Journal, 255, 716

6. Jokipii, J. R. 1987, The Astrophysical Journal, 313, 842

7. Giacalone, J. 2005, The Astrophysical Journal, 624, 765

8. Giacalone, J. 2005, The Astrophysical Journal, 628, L37

9. Jokipii, J. R., \& Giacalone, J. 2007, The Astrophysical Journal, 660, 336

10. Guo, F., \& Giacalone, J. 2010, The Astrophysical Journal, 715, 406

11. Guo, F., \& Giacalone, J. 2012, The Astrophysical Journal, 753, 28

12. Burgess, D. 2006, The Astrophysical Journal, 653, 316

13. Lu, Q., Hu, Q., \& Zank, G. P. 2009, The Astrophysical Journal, 706, 687 